\title{
Ultra-miniature eddy-current converter for small defects of deep occurrence search in the area of high-yield-stress steels weld joints
}

\author{
Vladimir Malikov ${ }^{1 *}$ and Alexey Ishkov $^{2}$ \\ ${ }^{1}$ Altay State University, 656049, Barnaul, Russia \\ ${ }^{2}$ Altay State Agricultural University, 656049, Barnaul, Russia
}

\begin{abstract}
The presented study describes a diagram of an eddy-current measuring system built on the basis of a subminiature eddy-current converter, intended for the conducting materials' study. The article describes the results of testing a designed measuring system for defects search in 6XB2S steel samples, connected by a weld joint. The technology for creating such materials permits the significant impact resistance of finished products and provides an optimal combination of hardness and toughness. However, the defects formed in the material near the weld joint become a serious problem in the operation of such materials. In this study, an assessment of the possibility of searching for model defects hidden by a weld in two steel plates was made. The defects had different linear dimensions $(0.3-0.9 \mathrm{~mm})$ and occurred at different depths $(1-2 \mathrm{~mm})$. The developed system showed high efficiency of scanning and searching for defects with linear dimensions up to $0.9 \mathrm{~mm}$. The search for smaller defects required a significant modernization of the developed measuring system - the introduction of a second eddy-current converter. The article presents the data confirming the feasibility of this change in the measuring system and the possibility of using a measuring system with two eddy current converters to search for small defects occurring at a considerable depth.
\end{abstract}

\section{Introduction}

A review of modern publications allows us to draw conclusions about the specifics of the use of steel grades in the manufacture of various devices and equipment [1-3].

So, in Russia and other countries a very limited list of structural carbon and alloy steels is used for these purposes, for example, $40-45,50 \mathrm{HG}(\mathrm{A}), 50-60 \mathrm{HG}, 35-65 \mathrm{G}$, as well as promising boron-containing steels $20-30 \mathrm{MnB} 5$ or its analogues. Instrumental steels of brands 40-50HF(A), 18-60HG(T), HC, HVG, 5-6HV2C have also found their niche. After passing through the heat treatment procedure, it is possible to achieve a surface hardness value of 4854 HRC for the first group of steels and 58-64 HRC for the second. Up to 70\% of cutting tools in mechanical engineering are made of high-speed steel, which includes refractory elements of tungsten and molybdenum.

\footnotetext{
${ }^{*}$ Corresponding author: osys11@gmail.com
} 
At the same time, an analysis of the actual literature over the past 5 years indicates the new trends in the practice of using steels. So, steel $65 \mathrm{G}$, a very common solution in the manufacture of working units of machines, is often replaced by boron steel 30MnB5. In turn, this grade can often be replaced by 5-6XV2S steel containing tungsten.

When choosing a material for specific purposes, it is important for the developers to take into account a set of parameters, since often an improvement in some consumer characteristics leads to a significant increase in post-processing requirements, and, ultimately, cost. So, the above-mentioned steels have special requirements for the heat treatment of metal: narrow temperature and wide time intervals of austenisation, which are combined with the increased requirements for this process temperature regime constancy (the heating temperature for hardening is about $800-900{ }^{\circ} \mathrm{C}$ ). All this ultimately leads to a twofold increase in the final material cost.

Steel $6 \mathrm{HV} 2 \mathrm{C}$ can be cited as a striking example. To obtain the maximum hardness, it is necessary to take into account its special sensitivity to thermal conditions, both during quenching and during tempering, while the duration of these processes can be up to 3 hours. In this case, after heat treatment, a soft layer of a substance that does not contain carbon 1 $\mathrm{mm}$ thick appears on its surface, which leads either to the need to use protective media or to additional mechanical treatment.

One of the most common types of defects in the manufacture of parts from steel 6XB2S is a defect in the form of cracks, leading to the destruction of the part under heavy loading [1]. One of the reasons for the cracks formation is the mechanical shock loads that the castings are subjected to during their transportation. In addition, often finished steel parts are additionally welded, after which it becomes extremely difficult to find defects in the weld area. Also, a crack in a steel part is a typical damage as a result of material degradation when used in aggressive environments. Such cracks can cause a key structural member to fail under normal operating loads and lead to severe accidents.

Non-destructive crack testing is essential to ensure the integrity of the parts and therefore the equipment safety. A popular method for searching for cracks in steel is ultrasonic testing [4-6]. However, the accuracy of crack sizing using this control method remains low, especially for shallow cracks in the metal.

Nondestructive eddy current testing remains one of the frequently used methods for searching the defects in electrically conductive materials $[7,8]$. This method has a number of advantages: high sensitivity to surface cracks, control efficiency, insensitivity to various contaminants and paint coatings on the surface of the test object, etc. However, the disadvantage of this test method is its inability to detect deep-seated defects [9]

Currently, due to this shortcoming of traditional eddy current testing methods $[10,11]$, the researchers in Russia and abroad such as D.G. Park and C. S. Angani from the Korea Atomic Energy Institute, S. Hosseini from Newcastle University [12-14], Z. Chen from Xi'an Jiaotong University and Z. Xu from Huazhong University of Science and Technology [15, 16] have improved eddy current testing technology, and have also proposed more and more new detection technologies.However, earlier researchers did not conduct systematic tests and experimental studies of weakly magnetic steel in the field of welds using eddy current testing technologies [17].

Thus, the development of an eddy current sensor that allows scanning steel for cracks, especially in the area of the weld, is still an urgent task.

The aim of the study was the development and testing of a measuring system that implements the eddy current method in its work and makes it possible to establish the presence of cracks in steel after welding. 


\section{Materials and methods}

The developed flaw detection system is based on an eddy current converter (ECC) overhead type, which includes three elements, namely: a coaxial generating inductance circuit, a coil that makes direct measurements and a special circuit capable of leveling the influence of the generating winding on the final measurement result.

The current of the generator winding of the converter, connected to the output of the generator, creates a two-frequency magnetic field. Due to the opposite connection of the measuring and compensation windings in the absence of an electrically conductive object near the converter, the output signal of the eddy-current converter turned out to be equal to zero. If there is an object of control near the converter, the magnetic field of excitation induces eddy currents of two frequencies in it. The magnetic field of these eddy currents produces an output signal (applied voltage) ECC. The analog conversion unit separates the amplitude components of the ECC signal, caused by each of the two frequency components of the eddy currents magnetic field. The output signals of the analog conversion block are proportional to the amplitudes of the ECC signals' real part high and low frequencies.

The output signals of the analog converter block are fed to the analog-to-digital converter, after which the data is transmitted to the data acquisition system, which registers the induced voltage on the receiving coil during the scanning process. Data processing and analysis system performs noise reduction and classification of detected signals.

The following parameters have been set for ECC: outer diameter of the excitation winding - $1 \mathrm{~mm}$; inner diameter of the excitation winding - $0.7 \mathrm{~mm}$; excitation winding height $-2 \mathrm{~mm}$; diameter of the middle turn of the measuring and compensation windings $-0.3 \mathrm{~mm}$; the distance between the planes of the middle turns of the measuring and compensation windings located symmetrically with respect to the excitation winding is $0.5 \mathrm{~mm}$. The excitation current frequency was 200 and $2000 \mathrm{~Hz}$. The frequencies were chosen so that, for the lower frequency, the penetration depth of the magnetic field into the test object was greater than the plate thickness values, for the upper frequency, the penetration depth into the metal was less than the plates thickness, but sufficient to ensure sensitivity to changes in the electrical conductivity of the material and the gap between ECC and the surface of the controlled object. The schematic of the manufactured eddy-current converter is shown in Fig. 1.

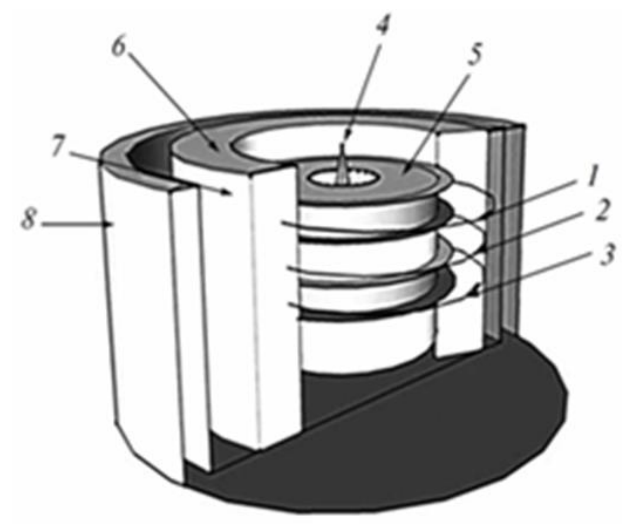

Fig. 1. Schematic diagram of an eddy-current converter. 1 - measuring winding, 2 - generation winding, 3 - compensation circuit 


\section{Research results}

To carry out an experimental study of the weld effect on the possibility of determining the defects of the plates, the plates of steel $6 \mathrm{XV} 2 \mathrm{~S}, 3 \mathrm{~mm}$ thick, connected by a weld, were made. The known model defects were contained at the edges of the plates to be joined. On plate No. 1 , before welding, flat-bottomed rectangular artificial defects with a width of $0.3,0.6,0.9$ $\mathrm{mm}$, a length of $5 \mathrm{~mm}$ and a depth of $1 \mathrm{~mm}$ were applied.

On plate No. 2, before welding, defects of the same thickness and length were applied, but the occurrence depth was $2 \mathrm{~mm}$. After the defect was deposited, both plates were subjected to electric arc welding in the defect area.

Scanning was carried out from the sample defect-free part side, across the weld in different areas. The amplitude of the signal at the exciting winding was $2 \mathrm{~V}$. Scanning was performed at a speed of $3 \mathrm{~mm} / \mathrm{s}$. The scanning scheme is shown in Fig. 2. Black arrows indicate the scanning direction of each defect.

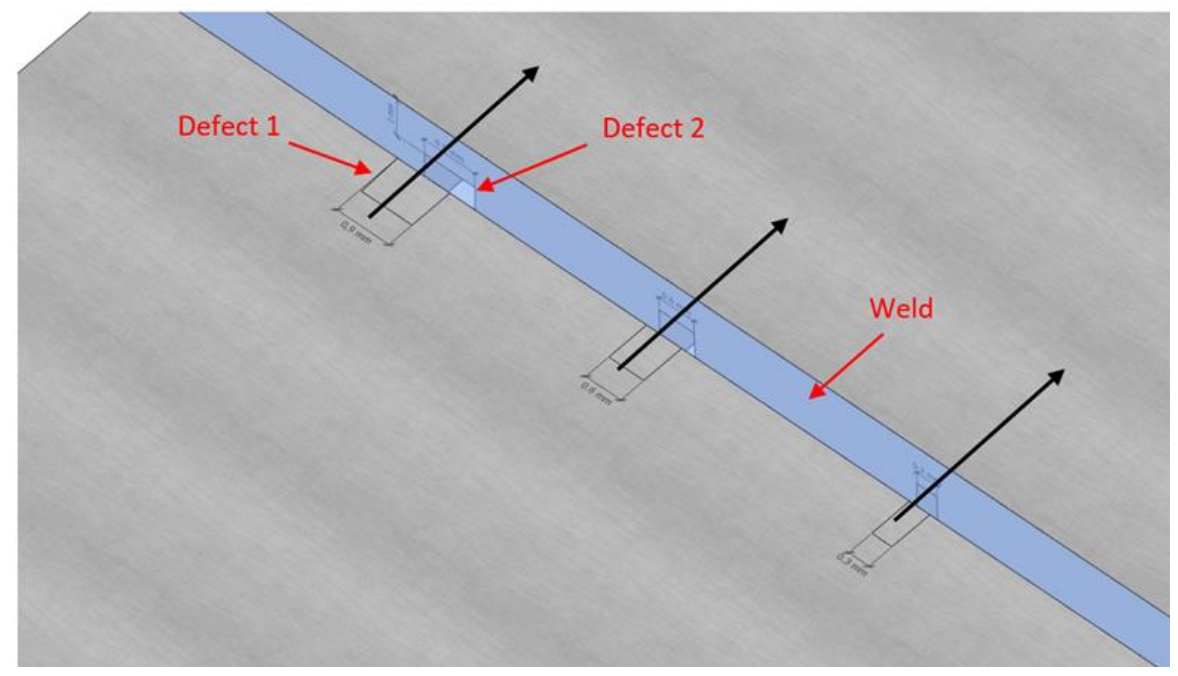

Fig. 2. Sample scanning scheme. Black arrows indicate scanning directions. Defect 1 - a defect located at a depth of $2 \mathrm{~mm}$. Defect 2 - a defect located at a depth of $1 \mathrm{~mm}$. Weld - weld area.

In the course of the experiments, the possibilities of using the developed eddy-current converter to search for defects lying in the plates next to the weld were found out.

Fig. 3 shows the results of scanning the sample in the area of defects $0.9 \mathrm{~mm}$ wide when operating with one eddy-current converter. The scanning results show a rather strong influence of the weld on the signal introduced into the measuring winding of the eddy-current converter. At a frequency of $2000 \mathrm{~Hz}$, when the magnetic field is concentrated on the surface of the test material, it is very difficult to separate the signal from the defects and the signal from the weld. A slight difference is visible only when analyzing the signal from defect No. 2, which lies at a depth of $1 \mathrm{~mm}$. When scanning at a frequency of $200 \mathrm{~Hz}$, the magnetic field penetrates deeper into the material under study and the difference between the signal from defect No. 1 (located at a depth of $2 \mathrm{~mm}$ ) and the signal from the weld is traced. However, on the contrary, the difference between the signal from the welded seam and the signal from defect No. 2, lying at a depth of $1 \mathrm{~mm}$, becomes less noticeable.

Fig. 4 shows the results of scanning defects with a width of $0.6 \mathrm{~mm}$ when working with one eddy-current converter. The scanning results when the sensor passes over the defects and over the weld seam are practically identical both at a frequency of $2000 \mathrm{~Hz}$ and at a frequency of $200 \mathrm{~Hz}$. It is extremely difficult to separate the signal from defects and the signal from the 
weld seam, and therefore the scan results are not practical for determining the defectiveness of the material under study.

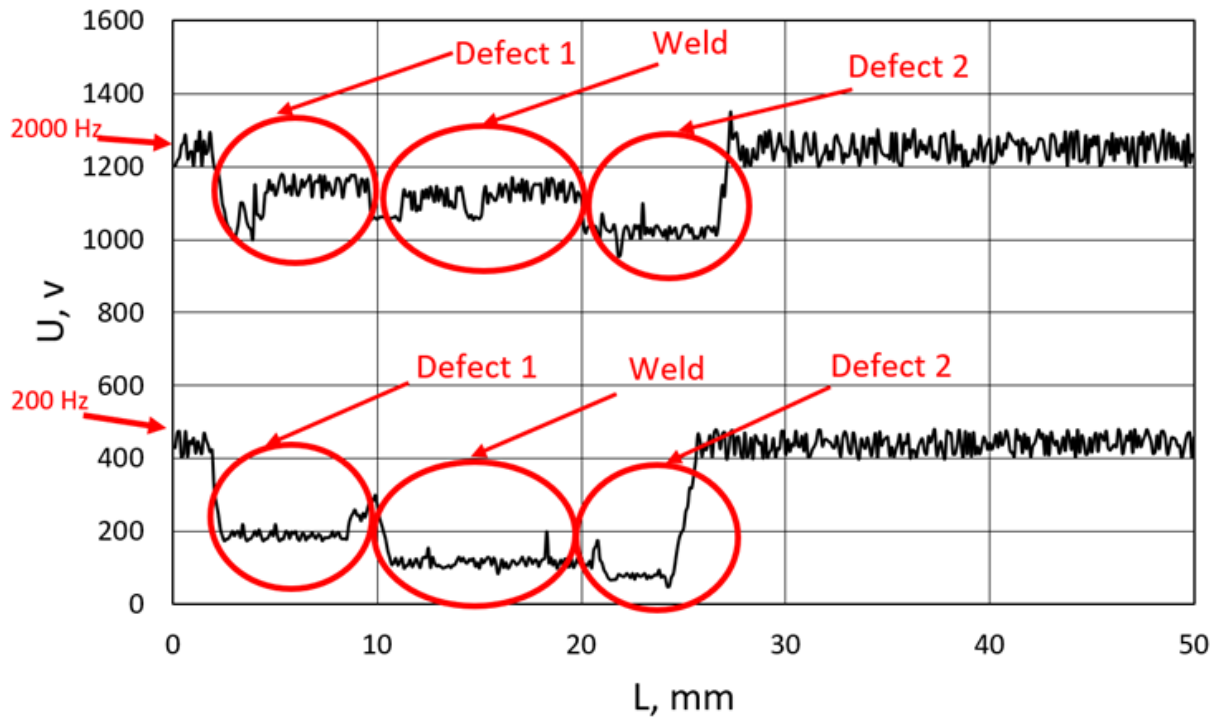

Fig. 3. Scheme of scanning a sample in an area with defects $0.9 \mathrm{~mm}$ wide using one eddy-current converter. $200 \mathrm{~Hz}$ - signal of the eddy-current converter when scanning at a frequency of $200 \mathrm{~Hz}, 2000$ $\mathrm{Hz}$ - signal of an eddy-current converter when scanning at a frequency of $2000 \mathrm{~Hz}$.

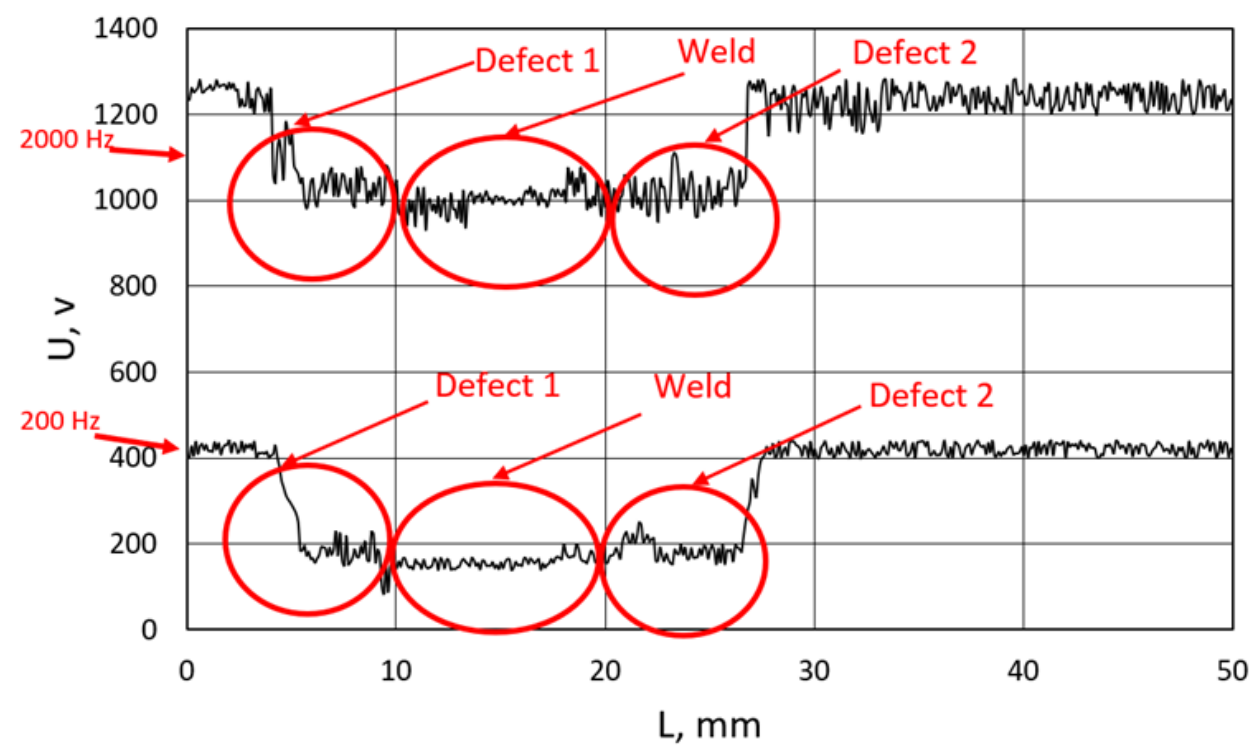

Fig. 4. Scheme of scanning a sample in a $0.6 \mathrm{~mm}$ wide area with defects using one eddy-current converter. $200 \mathrm{~Hz}$ - signal of the eddy-current converter when scanning at a frequency of $200 \mathrm{~Hz}, 2000$ $\mathrm{Hz}$ - signal of an eddy-current converter when scanning at a frequency of $2000 \mathrm{~Hz}$.

Due to the low quality of the results obtained when scanning defects less than $0.9 \mathrm{~mm}$, the developed measuring system was modernized. An additional eddy-current converter was included in the system opposite the first, so that the resulting signal was the difference between the signals of two converters. Such a switching scheme makes it possible to 
eliminate the influence of interfering factors, which, in this experiment, included the signal from the weld. For this, the second eddy current converter was located directly above the weld and did not move during scanning. The scanning was carried out as before with the first eddy converter. The scan results are shown in Fig. 5.

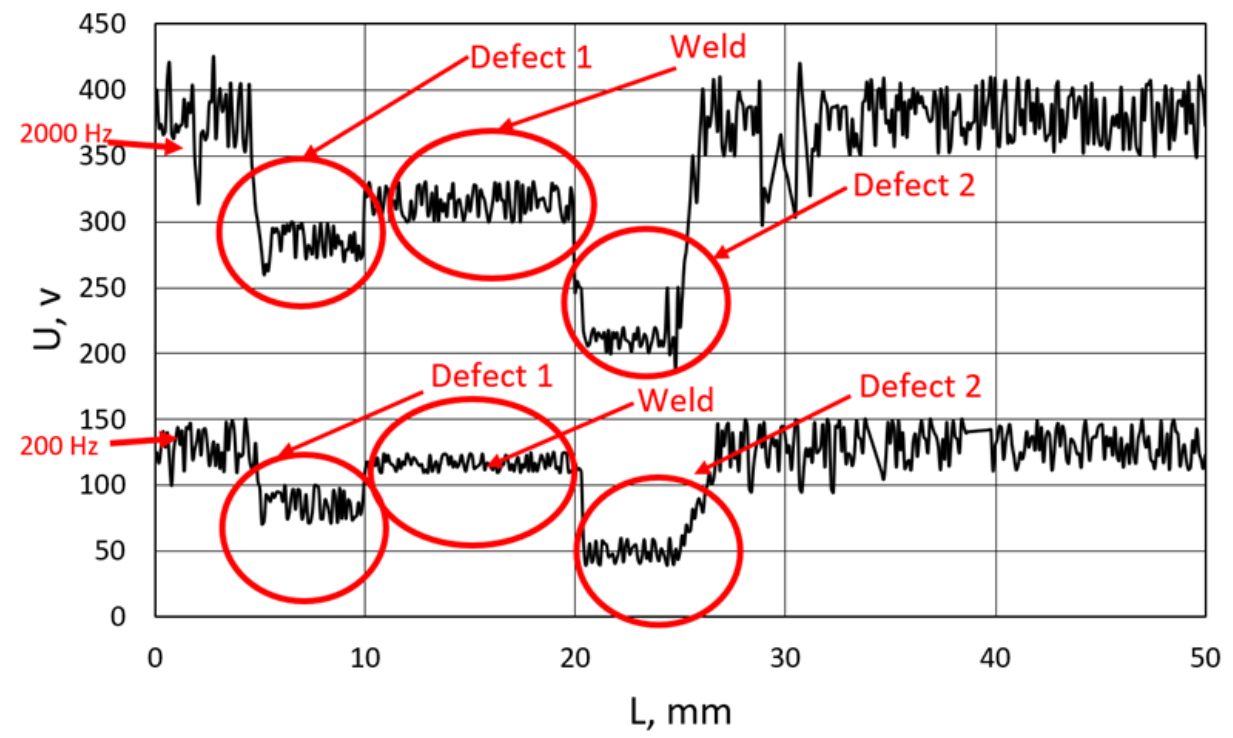

Fig. 5. Scheme of scanning a sample in a $0.6 \mathrm{~mm}$ wide area with defects using two eddy current converters. $200 \mathrm{~Hz}$ - signal of the eddy-current converter when scanning at a frequency of $200 \mathrm{~Hz}, 2000$ $\mathrm{Hz}$ - signal of an eddy-current converter when scanning at a frequency of $2000 \mathrm{~Hz}$.

When analyzing the scanning results obtained using one eddy-current converter, attention is immediately drawn to a significant decrease in the output signal amplitude of the measuring system. When using one converter in the system, the signal amplitude from the defect-free area was approximately $1200 \mathrm{mV}$ and $400 \mathrm{mV}$ for $2000 \mathrm{~Hz}$ and $200 \mathrm{~Hz}$ signals, respectively (Fig. 3, 4). When connecting the counter-second eddy-current converter, the signal amplitude from the defect-free area significantly decreased and amounted to $400 \mathrm{mV}$ and $140 \mathrm{mV}$ for the signals of $2000 \mathrm{~Hz}$ and $200 \mathrm{~Hz}$, respectively. This is due to the subtraction of two signals and, without prior adjustment, the decrease in the signal amplitude was even more significant. Therefore, during the connection of the second eddy-current converter, compensation for the decrease in the signal amplitude was performed using an additional trimming resistor in order to increase the scanning accuracy.

Analysis of the dependences obtained when scanning the test object using two eddy current converters shows a significant improved ability to search for defects and separate signals from defects with a width of $0.6 \mathrm{~mm}$ and a weld. Defects 1 and 2 when scanning both at a frequency of $200 \mathrm{~Hz}$ and at a frequency of $2000 \mathrm{~Hz}$ are clearly visible by a significant drop in the signal amplitude even against the background of the signal from the weld. The drop in the signal amplitude is from 30 to 50 percent, depending on the defect depth. While the drop in the signal amplitude when the converter passes over the weld is 10 to 20 percent relative to the signal from the defect-free part of the sample.

Fig. 6 shows the results of scanning the sample in the area of defects $0.3 \mathrm{~mm}$ wide. 


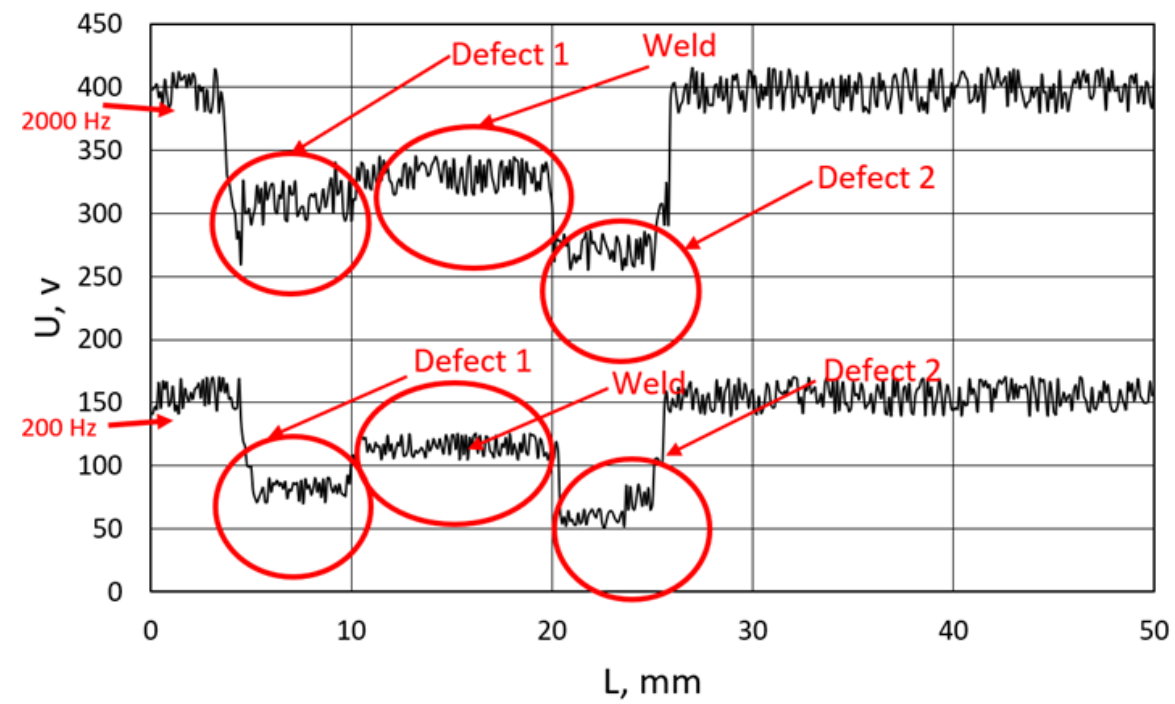

Fig. 6. Scheme of scanning a sample in an area with defects $0.3 \mathrm{~mm}$ wide using two eddy current converters. $200 \mathrm{~Hz}$ - signal of the eddy-current converter when scanning at a frequency of $200 \mathrm{~Hz}, 2000$ $\mathrm{Hz}$ - signal of an eddy-current converter when scanning at a frequency of $2000 \mathrm{~Hz}$.

The analysis of the obtained dependences when scanning a test object with defects having a width of $0.3 \mathrm{~mm}$ using two eddy current converters still demonstrates the effectiveness of the developed measuring system when searching for metal defects near the weld. Defect 2, located at a depth of $1 \mathrm{~mm}$, is quite clearly visible when scanning at a frequency of $2000 \mathrm{~Hz}$. The change in signal amplitude was about 35 percent compared to the signal level from the defect-free area. For comparison, the change in signal amplitude when scanning a weld was about 20 percent. Scanning the defect 1 at a frequency of $2000 \mathrm{~Hz}$ did not show a noticeable change in the signal level compared to the signal level from the weld, but good results were shown by scanning at a frequency of $200 \mathrm{~Hz}$. Both defects stand out noticeably against the background of the welded seam, demonstrating a drop in the signal amplitude by 40 and 60 percent from defects 1 and 2, respectively, with a drop in the signal level in the weld seam by 25 percent.

\section{Conclusion}

The developed measuring system has shown significant efficiency in scanning steel samples with the defects located in the weld area. The version of the measuring system with one eddycurrent converter provides search for defects with sizes from $0.9 \mathrm{~mm}$ and located at a depth of up to $2 \mathrm{~mm}$. A further decrease in the linear dimensions of defects and an increase in the depth of defects occurrence do not allow the use of this variant of the measuring system, which requires its significant modernization. The introduction of the second eddy-current converter and its installation on the weld seam permit the weld seam signal subtraction from the final signal of the measuring system, which provides significantly improved capabilities for finding defects. The modernized measuring system provides the ability to search for defects with linear dimensions from $0.3 \mathrm{~mm}$ and lying at a depth of up to $2 \mathrm{~mm}$. 


\section{References}

1. S.K. Alias, M.N. Halmy, M.A. Shah, IOP Conf. Series: Materials Science and Engineering 834, (2020) 012058.

2. Z. Wen, K. Dejun, International Journal of Fatigue 131, (2020) 105359.

3. Y.J. Zhou, Y. Zhang, F.J. Wang, Journal of Alloys and Compounds 466 (1-2), 201-204 (2008)

4. F. Hernandez-Valle, A.R. Clough, R. S. Edwards, Corrosion Science 78, 335-342 (2014)

5. I. Komura, T. Hirasawa, S. Nagai, Nuclear Engineering and Design 206, 351-362 (2001)

6. W. Cai, C. Jomdecha, Y. Zhao, L. Wang, Z. Chen, Philosophical Transactions of the Royal Society A Mathematical Physical and Engineering Sciences 378, 20190589 (2020)

7. G. Liu, Nondestructive Testing Technology (National Defense Industry Press, Beijing, 2006)

8. K. Xu, J. Zhou, Eddy Current Testing (Mechanical Industry Press, Beijing, 2004)

9. $\quad$ N. Yusa, L. Janousek, M. Rebican, N. Dohi, Nuclear Engineering and Design 236, 211221 (2006)

10. L. Yang, X. Guo, S. Gao, Instrument Technique and Sensor 10, 78-81 (2014)

11. H. Jiang, Research on Multi-frequency Eddy Current Testing Based on Frequency Modulated Excitation (Nanjing University of Aeronautics and Astronautics, Nanjing, 2013)

12. D.G. Park, IEEE transactions on magnetics 10, 3893-3896 (2009)

13. C.S. Angani, Nondestructive Evaluation 29, 248-252 (2010)

14. S. Hosseini, A. Lakis, NDT\&E International 47, 70-79 (2012)

15. Z. Chen, S. Xie, Electromagnetic Field Problems and Applications 39, (2012)

16. Z. Xu, X. Wu, C. Huang, Journal of Huazhong University of Science and Technology (Natural Science Edition) 6, 91-95 (2011)

17. Y. Liu, IOP Conference Series: Earth and Environmental Science 585, 012120 (2020) 